\title{
Physics of Tightly Curved Semiflexible Polymer Chains
}

\author{
Theo Odijk \\ Faculty of Chemical Engineering and Materials Science, Delft University of Technology, \\ P.O. Box 5045, 2600 GA Delft, The Netherlands
}

Received August 11, 1993; Revised Manuscript Received September 30, $1993^{\circ}$

\begin{abstract}
A deflection length determines the statistical physics of a tightly bent wormlike chain because the undulations of the chain must follow its "classical" path. The deflection length which is proportional to the square of the radius of the classical path and inversely proportional to the persistence length, is interpreted to be a critical scale. By comparing it to the usual deflection length for a wormlike chain confined by various forces, we can distinguish regimes of weak and strong bending. A scaling analysis is given of various problems: DNA packed in tori and phages, worms wound around cylinders, adsorption on spheres, hairpin and disclination defects in polymer nematics.
\end{abstract}

\section{Introduction}

Stiff chains are strongly bent in a surprising variety of systems of considerable practical importance. Doublestranded DNA is a case in point. In cells, a DNA molecule seldom undulates freely under the influence of thermal motion for it is often wrapped around the core particles within chromatin. ${ }^{1}$ In crystals, long DNA may fold ${ }^{2}$ or bend fairly sharply around defects. ${ }^{3}$ In bacteriophages ${ }^{4,5}$ and condensed structures, ${ }^{6-8}$ DNA presumably forms a tightly packed liquid crystal. An important theoretical issue is then the following. In dilute solution, a semiflexible chain possesses a considerable number of degrees of freedom. We wish to know how these are increasingly frozen out as we fold the chain. Under strong deformations, one expects a tightly bent chain to behave almost like a similarly folded elastic rod unperturbed by thermal motion. The purpose of this paper is to formulate the physics of this regime of tight bending.

Yamakawa and Stockmayer ${ }^{9}$ were the first to address the way degrees of freedom may be lost when a short stiff chain is coiled tightly into a circle of radius less than the persistence length $P$. However, precise numerical calculations of the statistical mechanics of sharply bent curves require mathematical ingenuity. ${ }^{9-12}$ Many problems involving interactions are even more complex so a qualitative picture should be valuable. Here, I present a scaling theory which has the drawback of lacking numerical coefficients.

The notion is to demarcate regions of weak and strong deformations. This can be simply illustrated by the folding of a cylindrical pore or tube of diameter $d(d \ll P)$ confining a semiflexible chain. If the tube is straight, the statistical properties of the worm are determined by the deflection length ${ }^{13} \lambda=d^{2 / 3} P^{1 / 3}$, the typical scale of chain collisions with the wall. If we bend the tube only slightly, $\lambda$ is not altered, at least to a first approximation. The collisions of the chain against the wall of the pore are not modified because the tube is relatively straight on the scale of the deflection length. The physics of such a slightly curved tube is quite analogous to that of a bent polymer nematic in which a chain may be viewed as a sequence of deflection segments aligned globally along the curved director. ${ }^{14,15}$ Ultimately, as the tube is bent too tightly, this perturbative picture must break down. I shall argue that it is possible to define a critical deflection length $\lambda_{c}$ which depends on the tube curvature: if $\lambda<\lambda_{c}$, the tube may be considered weakly bent; if $\lambda>\lambda_{c}$, a new regime 1993.

Abstract published in Advance ACS Abstracts, November 1,

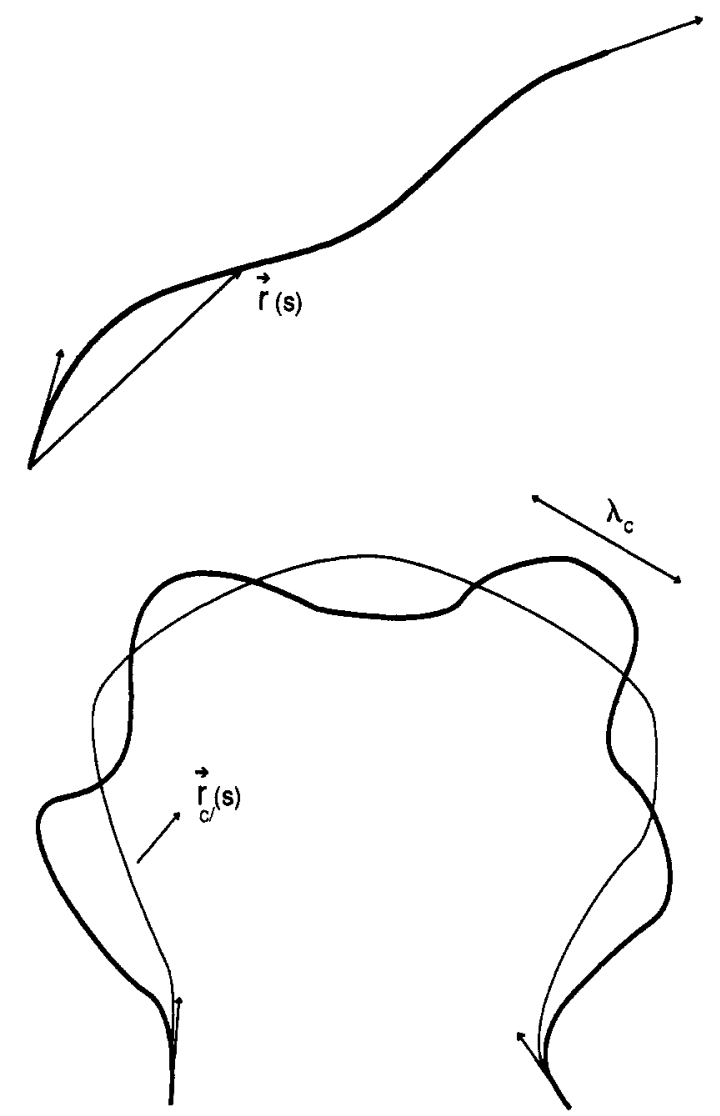

Figure 1. Wormlike chain with ends restrained: (a, top) weakly bent; (b, bottom) tightly bent but undulating close to the classical part $\vec{R}_{\mathrm{c} \mid}(s)$.

appears and the chain deflection length equals $\lambda_{c}$ instead of $\lambda$. In the latter case, the worm may be said to be tightly curved.

\section{Critical Deflection Length}

Let us bend an initially unconfined worm whose contour length $L$ is somewhat smaller than the persistence length $P$. The chain is at first kept in a deformed state by constraining the orientations of the tangential vectors at the two ends (Figure 1a). A configuration of the chain is described by the radius vector $\vec{r}(s)$ or the unit tangent vector $\vec{\mu}(s)=\partial \vec{r} / \partial s$ defined at each contour point $s(0 \leq$ $s \leq L)$. We may split $\vec{r}(s)$ into the classical path and the fluctuation from it

$$
\vec{r}(s)=\vec{r}_{\mathrm{c}}(s)+\delta \vec{r}(s)
$$




$$
\vec{\mu}(s)=\vec{\mu}_{\mathrm{c}}(s)+\delta \vec{\mu}(s)
$$

The bending energy of the chain is a minimum when it follows the classical path. It is well-known that for smalldeformations i.e. when the bending angles are small $\mid\left(\vec{\mu}_{\mathrm{c}}(L)-\vec{\mu}_{\mathrm{c} \mid}(0)|\ll 1,| \delta \vec{\mu} \mid \ll 1\right)$, we may approximate the bending energy harmonically. ${ }^{45}$ Then, the statistical mechanics becomes straightforward (see ref 16 and the Appendix).

At strong deformations, the harmonic approximation ${ }^{45}$ is no longer possible. ${ }^{9,10}$ Certain chain undulations which existed in the harmonic approximation are now frozen out (see Figure 1b; we now employ the additional constraint of the two ends being fixed at a small separation). I propose a scaling argument to show how this happens. In Figure $1 \mathrm{~b}$, the deflection $\delta_{c \mid}$ of the classical path with respect to the tangent vector at some point $s_{0}$ can be approximated by

$$
\delta_{\mathrm{c} \mid}\left(s-s_{\mathrm{O}}\right) \simeq \frac{\left(\left|s-s_{\mathrm{O}}\right|\right)^{2}}{R\left(s_{\mathrm{O}}\right)}
$$

It is noted that the radius of curvature $R$ of the path $\vec{r}_{\mathrm{c}}$ varies only mildly along the entire contour. If the worm were unrestrained and were to start at $\vec{\mu}_{\mathrm{c}}\left(s_{0}\right)$, we would have $^{13}$

$$
\left\langle\left(\delta \vec{r}(s)-\delta \vec{r}\left(s_{0}\right)\right)^{2}\right\rangle^{1 / 2} \text { undeformed } \simeq \frac{\left(\left|s-s_{0}\right|\right)^{3 / 2}}{P^{1 / 2}}
$$

Here, 〈〉 denotes an average over all configurations. Recall that the orientational fluctuation $\delta \vec{\mu}(s)$ is a Gaussian random process for $\left|s-s_{0}\right| \ll P$ when $|\delta \vec{\mu}| \ll 1$. Equations 2 and 3 allude to a new scale along the contour

$$
\lambda_{\mathrm{c}}=R^{2} / P \quad R<P
$$

This deflection length is interpreted as follows. For $\mid s-$ $s_{0} \mid<\lambda_{c}$, the chain can undulate quite freely close to the classical path (the initial orientation is now allowed to deviate a bit from $\left.\vec{\mu}_{\mathrm{c}}\left(s_{0}\right)\right)$. Then, eq 3 is a legitimate approximation. For $\left|s-s_{0}\right|>\lambda_{c}$, the chain must be deflected toward the classical path. In that case, eq 3 is no longer compatible, on average, with a typical real trajectory $\vec{r}_{\mathrm{c}}(s)+\delta \vec{r}(s)$ since $\delta_{\mathrm{c}}$ increases too fast with $s$. I conclude that $\lambda_{c}$ is a relevant scale: the chain undulates with a characteristic wavelength $\lambda_{c}$ along the classical path $\vec{r}_{\mathrm{c} \mid}(s)$ (see Figure 1b). Undulations of long wavelengths are progressively frozen out fast as the loop is tightened. Note that $\lambda_{c} \ll R$ whenever $R \ll P$. Also, the deflection length tends to $P$ when the radius of curvature does the same. This is what one expects: in the weakly bending or harmonic approximation, ${ }^{45}$ undulations of all wavelengths including $P$ are present but it is valid only if $R>$ $P$. Thus, eq 4 crosses over smoothly to the regime of weak bending. In general, we have $\lambda_{c}(s)=R^{2}(s) / P$ because often $\mathrm{d} R / \mathrm{d} s=0(1)$. A formal similarity transformation of the bending Hamiltonian agrees with these intuitive arguments (see the Appendix).

The free energy of a worm bent into a tight, uniform curve, is given by

$$
\begin{aligned}
F / k_{\mathrm{B}} T & \simeq \frac{L}{\lambda_{\mathrm{c}}}\left(1+\frac{\lambda_{\mathrm{c}}{ }^{2}}{R^{2}}\right) \\
& \simeq \frac{L P}{R^{2}}+\frac{L}{P} \quad R<P
\end{aligned}
$$

where $k_{\mathrm{B}}$ is Boltzmann's constant and $T$ is the temperature. This is derived by noting that: (a) the deflection length

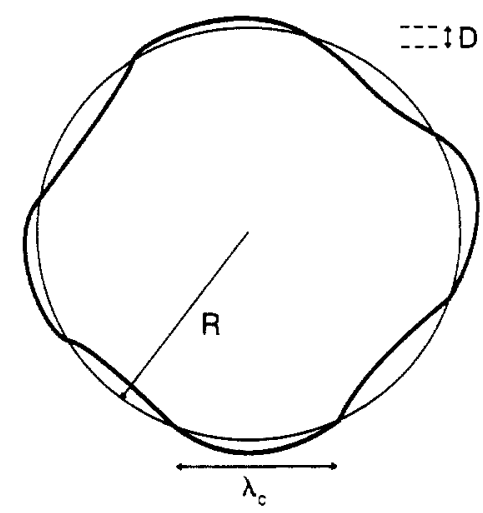

Figure 2. Fuzzy wormlike loop; $\lambda=R^{2} / P ; D=R^{3} / P^{2}$.

is the relevant scale for the undulations; (b) if $L \gg \lambda_{c}$, the free energy must be extensive, i.e. proportional to $L$; (c) the curvature is a vector so the next higher order term must be of relative magnitude $\lambda_{c}{ }^{2} / R^{2}$. The first term in eq 5 is simply the pure bending energy of a chain unperturbed by thermal motion. ${ }^{43}$ Hence, the scaling prescription is consistent, for this is what we expect in the limit of strong deformations. The second term expresses the relatively minor explicit influence of the undulations. ${ }^{44}$ For a circular, wormlike loop we have upon deleting a large numerical coefficient

$$
F_{\text {loop }} / k_{\mathrm{B}} T \simeq \frac{P}{L}+\frac{L}{P} \quad P<L
$$

This form agrees with the argument of the exponential term $^{42}$ in eq 45 of Shimada and Yamakawa. ${ }^{10}$ Because of the undulations, such a loop has a "fuzzy" thickness $D$ (Figure 2). The orientational fluctuations can be written as $\left\langle\theta^{2}\right\rangle \simeq D^{2} / \lambda_{c}{ }^{2} \simeq \lambda_{c} / P$ so that

$$
D \simeq R^{3} / P^{2}
$$

Under physiological conditions, a 40-nm-wide loop of DNA with a persistence length $P$ of $50 \mathrm{~nm}$ has a deflection length of $8 \mathrm{~nm}$ and the thickness of the "thermal" torus or "fuzzy" loop is about $5 \mathrm{~nm}$ ( $=D+$ diameter of the DNA helix).

I next adduce arguments for regarding $\lambda_{c}$ as a critical deflection length. At first, various external forces acting on a wormlike chain may balance in such a way that the worm is effectively confined within a thin straight tube. Suppose the fluctuations of the worm about the tube axis may be approximated harmonically, so that the second moment $\left\langle\delta \vec{r}^{2}\right\rangle=d^{2}$ is sufficient to understand its statistical properties $(d \ll P)$. The deflection length is simply ${ }^{13}$

$$
\lambda=d^{2 / 3} P^{1 / 3}
$$

As I pointed out in the Introduction, we already know $\lambda$ is almost unperturbed when the system is put under stress and the tube is bent weakly. In this case, we have $\lambda<\lambda_{\text {c }}$. Upon an increase in stress, the tube becomes progressively more curved - the tube radius of curvature $R_{\mathrm{t}}$ decreases until a line of reasoning similar to the above applies. Hence, we may write $\delta\left(\lambda_{c}\right) \simeq \lambda_{c}{ }^{2} / R_{\mathrm{t}} \simeq \delta_{\mathrm{c} \mid}\left(\lambda_{c}\right) \simeq \lambda_{c}{ }^{2} / R \simeq\left\langle\delta \vec{r}^{2}\right\rangle^{1 / 2}$ $=d$ in view of the classical path coinciding more or less with the deformed tube axis as soon as the regime of tight bending is entered; eq 4 shows that $\lambda_{c}$ is then equal to the deflection length $\lambda$ given by eq 8 . When the effective tube is bent even more, the deflection length must decrease if the path fluctuations are to conform to eq 2 for $|s-s o|>$ $\lambda$. In summary, we have

$$
\begin{array}{lll}
\lambda_{\text {eff }} \simeq \lambda & \text { if } & \lambda<\lambda_{c} \\
\lambda_{\text {eff }} \simeq \lambda_{c} & \text { if } & \lambda \geq \lambda_{c}
\end{array}
$$




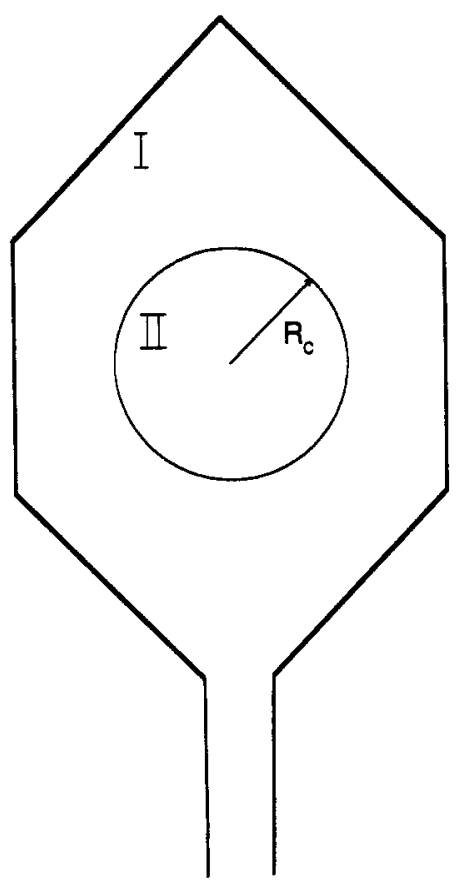

Figure 3. Bacteriophage filled with DNA. In the outer region (I), the hexagonal DNA lattice is deformed slightly. In the core (II), DNA is tightly packed.

and the free energy of the deformed worm is

$$
F / k_{\mathrm{B}} T \simeq \frac{L}{\lambda_{\text {eff }}}\left[1+\frac{\lambda_{\text {eff }}^{2}}{R_{\mathrm{t}}^{2}}\right]
$$

When $\lambda<\lambda_{c}$, the chain statistics is governed by thermally excited undulations competing with the confining force field; when $\lambda \geq \lambda_{c}$, the chain is a bent rod virtually unperturbed by neither. ${ }^{43}$

The application of eq 9 may be illustrated for DNA packed in a bacteriophage ${ }^{4,5}$ or torus. ${ }^{7}$ It is assumed that the helix is free of kinks. Because of the strong electrostatic interactions, the chain undulations in hexagonally packed DNA are quite small ${ }^{17,18}$ (at physiological ionic strengths, $d \simeq 0.5 \mathrm{~nm}, \lambda \simeq 2.3 \mathrm{~nm}$ ). From eqs 4 and 9 we calculate a critical radius of curvature $R_{\mathrm{c}} \simeq 11 \mathrm{~nm}$ corresponding to $\lambda=\lambda_{c}$. Hence, in the outer region (radius of curvature $\geq 11 \mathrm{~nm}$; origin in the middle of the phage, presumably), we may view the DNA lattice as a slightly deformed liquid crystal with undulations determined by $d$ or $\lambda$. In the core region $(R \leq 11 \mathrm{~nm})$, the DNA is essentially a bundle of tight elastic loops which does not retain the physical properties of the surrounding liquid crystal (Figure 3). Within the core, the free energy per unit length increases dramatically with the curvature, but it is almost constant in the outer region. Thus, if such a tightly bent inner region exists, it exists at high cost. The exact structure of DNA in phages is still debated. ${ }^{5}$ Similar reasoning applies to toroidal DNA complexes. One suspects that the minimum value $R_{\mathrm{m}}$ of the inner toroidal radius must somehow be connected with $R_{\mathrm{c}}$. Indeed, $R_{\mathrm{c}}$ is quite close to $R_{\mathrm{m}} \simeq 9 \mathrm{~nm}$ found in recent experiments by Arscott et al. ${ }^{7}$ Evidently, these problems merit a more quantitative analysis (see also ref 19). I next survey several more consequences of eq 9 .

\section{Chain under Tension Wound around a Cylinder}

First, I discuss the unwound case. A scaling analysis of a flexible chain under strong tension was given by Pincus ${ }^{20}$ who regarded the chain as a sequence of blobs each having a size inversely proportional to the tensile force $\vec{f}$. Naive

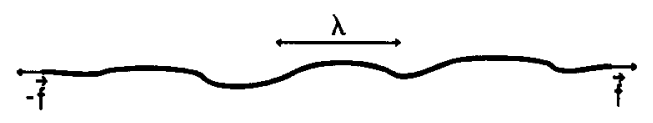

(a)

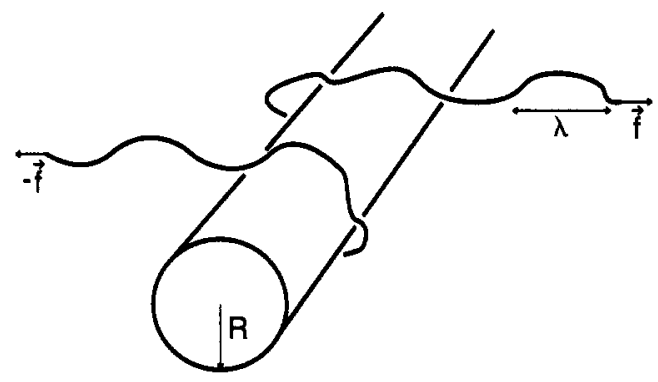

(b)

Figure 4. (a) Wormlike chain under tension. (b) Strained worm wrapped once around a cylinder.

application to a strongly stretched worm would then give

$$
f \simeq k_{\mathrm{B}} T / \lambda \quad \text { (incorrect) }
$$

In effect, the argument would run that the deflection length is simply the sole relevant scale and eq 11 is the result of dimensional analysis (see Figure 4). But in the Pincus analysis ${ }^{20}$ the blobs are isotropic units whereas the deflection blobs or segments experience a torque and reorient. Accordingly, the orientational fluctuations are bound to be important so a more careful line of reasoning is required.

The free energy of the wormlike chain is enhanced by the tensile force

$$
\begin{aligned}
\Delta F & =-\langle(\vec{r}(L)-\vec{r}(0)) \cdot \vec{f}\rangle \\
& =\int_{0}^{L}\langle\vec{\mu}(s) \cdot \vec{f}\rangle \mathrm{d} s \\
& =-f L \int_{0}^{L}\langle\cos \theta(s)\rangle \mathrm{d} s \\
& \simeq \text { constant }+1 /{ }_{2} f L\left\langle\theta^{2}\right\rangle
\end{aligned}
$$

where $\theta(s)$ is the angle between the force vector and the tangent vector $\vec{\mu}(s)$. The last equality is valid because the tension is strong. The chain is effectively $L / \lambda$ deflection segments, each acting in the main like an independent unit (Figure 4). Therefore, by equipartition we have

$$
f \lambda\left\langle\theta^{2}\right\rangle \simeq k_{\mathrm{B}} T
$$

Now, the inner region of a deflection segment is not influenced by the external force, to first order, which means the orientational correlation is the usual Gaussian random process $^{16}\left(\left(\theta\left(s_{1}\right)-\theta\left(s_{2}\right)\right)^{2}\right\rangle \simeq\left|s_{1}-s_{2}\right| / P$ for $s_{1}$ and $s_{2}$ on the inner contour. Extending this to its outer limit of validity, we get

$$
\left\langle\theta^{2}\right\rangle \simeq 1 /{ }_{2}\left\langle(\theta(0)-\theta(\lambda))^{2}\right\rangle \simeq \lambda / P
$$

Finally, eliminating the angle, we obtain

$$
\lambda^{2} \simeq k_{\mathrm{B}} T P / f \quad \lambda<P
$$

Next, the semiflexible chain is wound only once around a cylinder of radius $R_{\text {cyl }}$ and then strained by a force $\vec{f}$. If the chain's trajectory were to follow the circumference of 
the cylinder, we would have $\lambda_{c}=R_{\text {cyl }}^{2} / P$. Hence, if $\lambda \gg \lambda_{c}$, the cylinder is effectively needlelike and nothing but a topological entanglement constraining the worm to have one loop of size $(\lambda P)^{1 / 2}=\left(k_{\mathrm{B}} T P^{3} / f\right)^{1 / 2}$. Steadily increasing the tension, we reach the limit $\lambda=\lambda_{c}$ when the chain can be said to envelop the cylinder, touching it about onceevery $\lambda_{c}$ (Figure 4). Equation 15 yields for the critical force

$$
f_{\mathrm{c}}=k_{\mathrm{B}} T P^{3} / R_{\mathrm{cyl}}{ }^{4}
$$

It increases very fast with decreasing cylinder radius. ${ }^{41}$ This calculation may bear on a situation where DNA is strained while it wraps around nucleosome particles.

\section{Adsorption on a Small Sphere}

Maggs et al. ${ }^{21}$ address the adsorption of a wormlike chain on a flat surface, both analytically and numerically. The attractive potential has a short range. They are able to prove that the binding is discontinuous for slightly undulating chains $(L<P)$. It is possible to show that ${ }^{46}$

$$
\lambda_{h} \Delta w \simeq k_{\mathrm{B}} T \quad \lambda_{\mathrm{h}}<P
$$

Here, $\Delta w=w-w_{0}, w$ is the binding energy per unit length of chain and $w_{\mathrm{O}}=O\left(k_{\mathrm{B}} T / P\right)$. The deflection length of a loop of height $h$ is given by

$$
\lambda_{h}=h^{2 / 3} P^{1 / 3} \quad \lambda_{h}<P
$$

The adsorbed chain is a wormlike sequence of $L / \lambda_{h}$ more or less identical loops.

It is clear that such a string of deflection loops bound to a sphere of radius $R_{\mathrm{g}}$ is impossible unless $\lambda_{h} \leq \lambda_{\mathrm{c}}=$ $R_{\mathrm{s}}{ }^{2} / P$. Accordingly, the new "critical" binding energy $w_{\mathrm{s}}$ can be expressed by

$$
\left(w_{\mathrm{s}}-w_{0}\right) / k_{\mathrm{B}} T \equiv \Delta w_{\mathrm{s}} / k_{\mathrm{B}} T \simeq P / R_{\mathrm{s}}^{2} \quad R_{\mathrm{s}}<P
$$

provided the range of the potential is much smaller than $R_{\mathrm{g}}$. The thickness of the bound layer is

$$
\begin{aligned}
h & \simeq\left(k_{\mathrm{B}} T / \Delta w\right)^{3 / 2} P^{-1 / 2} \\
& \simeq \frac{R_{\mathrm{s}}^{3}}{P^{2}}\left(\frac{\Delta w_{\mathrm{s}}}{\Delta w}\right)^{3 / 2} \quad \Delta w \geq \Delta w_{\mathrm{s}}
\end{aligned}
$$

At the binding transition, it turns out that the wandering of a worm on a sphere with $R_{\mathrm{s}}<1 /{ }_{3} P$ is quite similar to that of a worm trapped on a flat surface. On the one hand, we have $\lambda_{\mathrm{s}}<1 / 3 R_{3}$. On the other, the transverse displacement is

$$
R_{\mathrm{T}} \simeq\left(L / \lambda_{\mathrm{c}}\right)^{1 / 2} h \simeq L^{1 / 2} R_{\mathrm{s}}{ }^{2} P^{-3 / 2}
$$

and $R_{\mathrm{T}}<R_{\mathrm{s}} / 6$ for $L<2 P$. Thus, the trajectory of such a chain is very close to a great circle of radius $R_{\mathrm{s}}$. We can also safely neglect stability problems of the kind discussed by Manning ${ }^{22-24}$ for relaxed lines on a curved surface. In the regime $1 /{ }_{3} P<R_{\mathrm{B}}<P$, we have tacitly assumed that the chain undulates along a great circle also. The wandering implied by eq 21 is somewhat greater, an effect that may modify eq 19, but it is outside the scope of this paper.

At the binding transition, $w=w_{\mathrm{s}}$, a longer chain ( $L \gg$ $P$ ) will tend to be wound around the sphere a few times. It will be reeled in very fast as $w$ increases (Figure 5). The

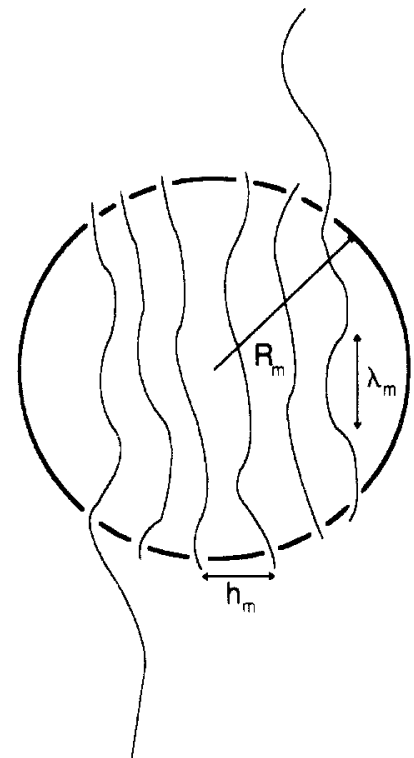

Figure 5. Long wormlike chain bound to a small sphere in uniformly thick winds.

minimum radius of curvature $R_{\mathrm{m}}$ is given by

$$
\Delta w / k_{\mathrm{B}} T \simeq \lambda_{\mathrm{m}}^{-1}=P / R_{\mathrm{m}}^{2}
$$

Since loops of the chain adsorbed elsewhere on the sphere have a critical deflection length $\lambda_{c}>\lambda_{m}$, the effectivedeflection length is $\lambda_{m}$ for the whole layer. Hence, we find for the number of winds $n$

$$
\begin{aligned}
n-1 & \simeq\left(\frac{R_{\mathrm{s}}{ }^{2}-R_{\mathrm{m}}{ }^{2}}{h_{\mathrm{m}}{ }^{2}}\right)^{1 / 2} \\
& \simeq \frac{P \Delta w}{k_{\mathrm{B}} T}\left(\frac{\Delta w}{\Delta w_{\mathrm{s}}}-1\right)^{1 / 2} \\
& \simeq \frac{P \epsilon}{k_{\mathrm{B}} T}
\end{aligned}
$$

It is concluded that a slight increment in the binding energy $\left(\epsilon \equiv w-w_{\mathrm{s}}\right.$ ) causes a large number of winds. This calculation neglects the excluded-volume interaction between adjacent loops. Tranverse wanderings given by eq 21 are frozen out as the chain is packed onto the sphere. But the free energy of interaction is at most of the same order of magnitude as the total binding free energy, so eq 23 remains a reasonable estimate. More importantly, the distance between neighboring winds can, of course, never be smaller than the chain diameter $a$ itself. Therefore, eq 23 does break down for $h_{\mathrm{m}}<a$.

There is another complication for very long chains. The transition described by eq 19 may have to be amended because of the adsorption of large loops of sizes much greater than the persistence length. Also, several spheres may be strung along the chain as in a pearl necklace. This is the case for histone octamers bound to linker DNA in chromatin, though the cores are not spheres but rather more like flat cylinders of the same height and radius of about $5 \mathrm{~nm}$. Unbinding of the DNA is known to occur at fairly high ionic strengths. ${ }^{25}$ As pointed out above, on a small sphere transverse undulations are almost negligible so that eqs 19 and 20 should be useful in understanding the winding of DNA around the circumference of a core particle. In fact, the slight transverse wandering is compatible with the helical winding of DNA. 


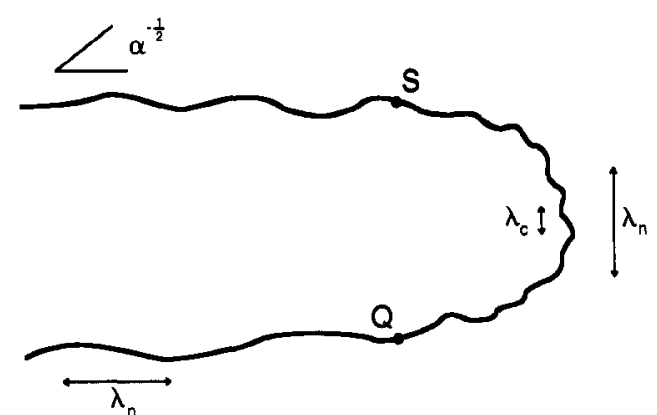

Figure 6. Hairpin in a nematic. The loop between $S$ and $Q$ defines a central region where the chain is bent tightly. At $S$ and $\mathrm{Q}$ the radius of curvature $R_{\mathrm{c}}=P / \alpha^{1 / 2}$.

\section{Hairpins in Polymer Nematics}

Clear evidence for the existence of chain hairpins in a thermotropic polymer nematic has been found only recently. ${ }^{26}$ First introduced independently by de Gennes ${ }^{27}$ and Khokhlov and Semenov, ${ }^{26}$ the mathematical theory of hairpin formation has progressed rapidly. ${ }^{11,29-34}$ In apolymer nematic, undulations are governed by the deflection length ${ }^{35}$

$$
\lambda_{\mathrm{n}}=P / \alpha
$$

where the large parameter $\alpha=2 /\left\langle\theta^{2}\right\rangle$ is a measure of the orientational order with respect to the director. Using eqs 4,9 , and 24 , we can formulate a critical radius of curvature for a test chain deformed within the nematic

$$
R_{\mathrm{c}} \simeq\left(\lambda_{\mathrm{n}} P\right)^{1 / 2} \simeq P / \alpha^{1 / 2}
$$

Thus, a hairpin can be visualized as in Figure 6. There is an inner curve which is bent tightly and whose maximum dimension must be $R_{\mathrm{c}}$. The two tails extending into the bulk basically have the statistical properties of a chain undulating along the director without folding.

However, the hairpin does not approximate a semicircle of radius $R_{\mathrm{c}}$ since it is sharpened by the nematic torque exerted by the nematic fluid of chains surrounding the defect. If the deflection length $\lambda_{n}$ is a relevant scale in the full Hamiltonian, 11,27,32,34 the inner region shown in Figure 6 must contain a tight semicircle of radius $\lambda_{n}$ which dominates in a calculation of the bending energy $(E \simeq$ $\alpha k_{\mathrm{B}} T$ ) of a hairpin. The undulations within the semicircle have a deflection length (Figure 6)

$$
\lambda_{\mathrm{c}} \simeq \lambda_{\mathrm{n}}{ }^{2} / P \simeq P / \alpha^{2}
$$

Such a minute scale also arises in a different scaling context discussed by Williams and Halperin. ${ }^{34}$

\section{$-1 / 2$ Wedge Disclination}

Another instance of a tightly elastic nonuniformity arises in a straight $-1 / 2$ wedge disclination in a polymer nematic. For this director field shown in Figure 7, the critical radius of curvature $R_{\mathrm{c}}$ is again given by eq 25 . Geometricalarguments prove that the size of the inner region is at most

$$
\begin{aligned}
W & \simeq\left(2.3^{-1 / 2}-1\right) R_{\mathrm{c}} \\
& \simeq 0.15 P / \alpha^{1 / 2} \\
& \simeq 0.15 P^{2 / 3} a^{1 / 3} \phi^{-1 / 3}
\end{aligned}
$$

where $a$ is the chain diameter. The last equality is valid for lyotropics ${ }^{35}$ (volume fraction $\phi \ll 1$ ), Equation 27 is conjectural, for the precise nature of the core region is not clear (tightly bent chains, biaxial or almost isotropic order, aggregating chain ends, ${ }^{36}$ etc).

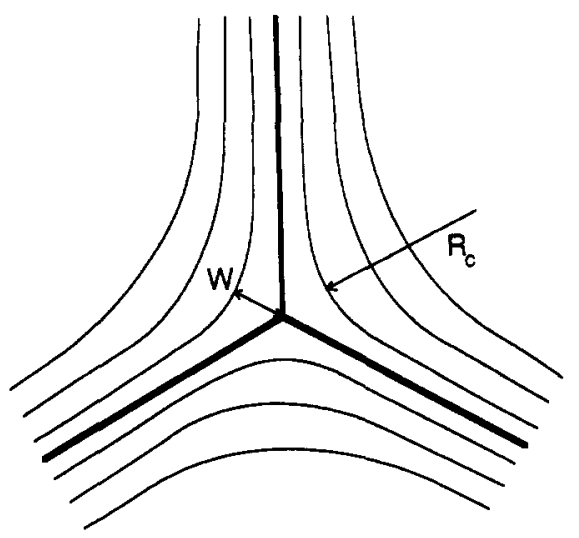

Figure 7. $-1 / 2$ wedge disclination in a polymer nematic.

An analogous breakdown of the nematic order should also occur for a suspension of rigid rods. When the director is strongly curved, the rods can no longer align along it, within the bounds of the orientation distribution which is sharply packed. The critical radius is

$$
\begin{gathered}
R_{\mathrm{c}} \simeq L \alpha^{-1 / 2} \\
W \simeq 0.15 R_{\mathrm{c}} \simeq 0.15 a / \phi
\end{gathered}
$$

Equation 29 is in qualitative agreement with the size of the core detected in Monte Carlo simulations of the $-1 / 2$ disclination in rod nematics. ${ }^{37}$

\section{Concluding Remarks}

The simple scheme proposed in eqs 4,9 , and 10 is useful for several reasons. It is now feasible to derive scaling expressions for phenomena involving semiflexible chains that are tightly curved. Up to a point, it becomes possible to understand the physics of complicated WKB analyses $^{9-12,38}$ which are not reducible to harmonic approximations. ${ }^{45}$ Finally, scaling analyses may provide a basis for devising efficient matching procedures in the numerical work itself.

\section{Appendix}

The bending Hamiltonian of the wormlike chain ${ }^{45}$ is given by

$$
H[\vec{r}(s)]={ }_{1}^{1} / 2 P \int_{0}^{L} \mathrm{~d} s\left(\partial^{2} \vec{r} / \partial s^{2}\right)^{2}
$$

and the partition function under suitable restraints at the two ends (e.g. $\vec{\mu}(0)$ and $\vec{\mu}(L)$ fixed) is

$$
Z=\int D[\vec{\mu}(s)] \mathrm{e}^{-H / k_{\mathrm{B}} T}
$$

This functional integral is constrained ${ }^{45}$ by the condition $\vec{\mu}^{2}(s)=1$. If we split $\vec{r}(s)$ into the classical path $\vec{r}_{\mathrm{c}}(s)$ and the fluctuation $\delta \vec{r}(s)$ from it, eq A1 separates into two terms

$$
H=H\left[\vec{r}_{\mathrm{c}}(s)\right]+H[\delta \vec{r}(s)]
$$

The harmonic approximation ${ }^{45}$ results in the regime of weak bending when the curvature vector $\vec{\rho}(s)=\partial^{2} \mathrm{r}_{\mathrm{c}} / \partial s^{2}$ of the classical path is small in magnitude. ${ }^{16}$ Then, $\mu(s)$ can be replaced by the small angle $\vec{\theta}(s)=\vec{\theta}_{\mathrm{c}}(s)+\delta \vec{\theta}(s)$ with respect to the fixed tangential vector $\mu(0)$, i.e. $\vec{\mu}(s) \simeq(\vec{\theta}(s)$, 1), so the partition function reduces to a standard Gaussian functional integral without restrictions. ${ }^{45}$

$$
\begin{aligned}
Z \simeq Z_{\text {harm }} & =\int D[\vec{\theta}(s)] \mathrm{e}^{-H[\vec{\theta}(s)] / k_{\mathrm{B}} T} \\
& =Z_{\text {cl }} \int D[\delta \vec{\theta}(s)] \mathrm{e}^{-H[b \vec{\theta}(s)] / k_{\mathrm{B}} T}
\end{aligned}
$$

For tight curves, the evaluation of functional integrals of 
the kind in eq A2 is very difficult. $10,38,39$ Nonetheless, a similarity transformation ${ }^{40}$ of eq A3 is straightforward. I introduce the following primed variables of order unity: $\vec{\rho}(s)=R^{-1} \vec{\rho}^{\prime}\left(s^{\prime}\right), s \equiv \Lambda s^{\prime}, L \equiv \Lambda L^{\prime}$ and $\delta \vec{r} \equiv \Delta \delta \vec{r}^{\prime}$.

$$
H=\frac{\Lambda P}{2 R^{2}} \int_{0}^{L^{\prime}} \mathrm{d} s^{\prime}\left[\left(\vec{\rho}^{\prime}\left(s^{\prime}\right)\right)^{2}+\frac{R^{2} \Delta^{2}}{\Lambda^{4}}\left(\frac{\partial^{2} \delta \vec{r}^{\prime}\left(s^{\prime}\right)}{\delta s^{\prime 2}}\right)^{2}\right]
$$

This Hamiltonian is only scale invariant if we choose $\Lambda \equiv$ $\lambda_{\mathrm{c}}$ (eq 4) and $\Delta \equiv D$ (eq 7),

$$
H=1 / 2 \int_{0}^{L^{\prime}} \mathrm{d} s^{\prime}\left[\left(\vec{\rho}^{\prime}\left(s^{\prime}\right)\right)^{2}+\left(\partial^{2} \delta r^{\prime}\left(s^{\prime}\right) / \partial s^{\prime 2}\right)^{2}\right]
$$

Accordingly, for a long chain we have

$$
F / k_{\mathrm{B}} T \simeq L^{\prime}=L / \lambda_{\mathrm{c}}
$$

because the free energy must be extensive.

\section{References and Notes}

(1) Van Holde, K. E. Chromatin; Springer: New York, 1989.

(2) Lerman, L. S.; Wilkerson, L. S.; Venable, J. H.; Robinson, B. H. J. Mol. Biol, 1976, 108, 271.

(3) Livolant, F. J. Mol. Biol. 1991, 218, 165.

(4) Earnshaw, W. C.; Harrison, S. C. Nature 1977, 268, 598.

(5) Lepault, J.; Dubochet, J.; Baschong, W.; Kellenberger, E. EMBO J. 1987, 6, 1507.

(6) Laemmli, U. K. Proc. Natl. Acad. Sci. U.S.A. 1975, 72, 4288.

(7) Arscott, P. G.; Li, A. Z.; Bloomfield, V. A. Biopolymers 1990, 30,619 .

(8) Plum, G. E.; Arscott, P. G.; Bloomfield, V. A. Biopolymers 1990 , 30,631 .

(9) Yamakawa, H.; Stockmayer, W. H. J. Chem. Phys. 1972, 57, 2843.

(10) Shimada, J.; Yamakawa, H. Macromolecules 1984, 17, 689.

(11) Vroege, G. J.; Odijk, T. Macromolecules 1988, 21, 2848.

(12) For an analogous surface problem - a wormhole passage in a lamellar phase-see: Goos, J.; Gompper, G. J. Phys. I 1993, 3, 1551.

(13) Odijk, T. Macromolecules 1983, 16, 1340. For computer simulations establishing the scaling and density distribution, see: Dijkstra, M.; Frenkel, D.; Lekkerkerker, H. N. W. Physica A 1993, 193, 374.

(14) Odijk, T. Liq. Cryst. 1986, 1, 553

(15) Grosberg, A. Yu.; Zhestkov, A. V. Polym. Sci. USSR 1986, 28, 97.

(16) Landau, L. D.; Lifshitz, E. M. Statistical Physics, 3rd ed.; Pergamon: Oxford, U.K. 1980; Part 1.

(17) Podgornik, R.; Rau, D. C.; Parsegian, V. A. Macromolecules $1989,22,1780$.

(18) Odijk, T. Biophys. Chem. 1993, 46, 69.

(19) Grosberg, A. Yu.; Zhestkov, A. V. J. Biomol. Struct. Dyn. 1986, $3,859$.

(20) Pincus, P. Macromolecules 1976, 9, 386.

(21) Maggs, A. C.; Huse, D. A.; Leibler, S. Europhys. Lett. 1989, 8, 615 .
(22) Manning, G. S. Q. Appl. Math. 1987, 45, 515.

(23) Manning, G. S. Q. Appl. Math. 1987, 45, 809

(24) Manning, G. S. J. Chem. Phys. 1992, 96, 4007.

(25) Oohara, I.; Wada, A. J. Mol. Biol. 1987, 196, 399.

(26) Li, M. H.; Brulet, A.; Davidson, P.; Keller, P.; Cotton, J. P. Phys. Rev. Lett. 1993, 70, 2297.

(27) de Gennes, P. G. In Polymer Liquid Cystals; Ciferri, A. Krigbaum, W. R., Meyer, R., Eds.; Academic Press: New York, 1982.

(28) Khokhlov, A. R.; Semenov, A. N. J. Phys. A. 1982, 15, 1361

(29) Warner, M.; Gunn, J. M. F.; Baumgärtner, A. B. J. Phys. A $1985,18,3007$

(30) Williams, D. R. M.; Warner, M. J. Phys. Fr. 1990, 51, 317.

(31) Gunn, J. M. F.; Warner, M. Phys. Rev. Lett. 1987, 58, 393.

(32) Kamien, R. D.; Le Doussal, P.; Nelson, D. R. Phys. Rev. A 1992, $45,8727$.

(33) Selinger, J. V.; Bruinsma, R. F. J. Phys. 1992, 2, 1215

(34) Williams, D. R. M.; Halperin, A. Macromolecules 1993, 26, 2025

(35) Odijk, T. Macromolecules 1986, 19, 2313.

(36) Kléman, M. In Liquid Crystallinity in Polymers; VCH Publishers: Cambridge, U.K., 1991.

(37) Hudson, S. D.; Larson, R. G. Phys. Rev. Lett. 1993, 70, 2916.

(38) For a good discussion of semiclassical methods near classical curved paths, see: Berry, M. V.; Mount, K. E. Rep. Prog. Phys. $1972,35,315$.

(39) Gutzwiller, M. C. J. Math. Phys. 1967, 8, 1979.

(40) Odijk, T. Macromolecules 1984, 17, 502

(41) The tension within a fuzzy torus (Figure 2) thus scales as $16 \pi^{4}$ $k_{\mathrm{B}} T P^{3} / L^{4}$ and is also considerable for $L \ll P$

(42) In ref 10 , Shimada and Yamakawa derive a certain Green function for a circular worm. Since this function is proportional to a probability which itself may be viewed as a Boltzmann factor associated with an effective free energy of curvature, we may compare eq 6 with the argument of the exponential in eq 45.

(43) This is quite analogous to what happens in the semiclassical limit of quantum mechanics: the classical path is independent of the de Broglie wavelength.

(44) This term may be viewed as the slight increase in free energy upon bending each deflection segment (more or less) into \& circular section of radius $R$.

(45) In spite of being quadratic, the bending Hamiltonian of eq Al may be termed anharmonic in view of the constraint $\mu^{2}(s)=$ $\mu_{x}^{2}(s)+\mu_{y}^{2}(s)+\mu_{z}^{2}(s)=1$. In general, anharmonic terms appear on rewriting eq $\mathrm{A} 1$ in terms of the truly independent variables $\mu_{x}(s)$ and $\mu_{y}(s)$. In the weakly bending approximation, the anharmonic terms may be disregarded so I call it the "harmonic approximation" in this paper. Note that a (constrained) quadratic Hamiltonian is often termed harmonic in the theoretical physics literature.

(46) Maggs et al. ${ }^{21}$ point out that, for a discrete worm confined between two walls and adsorbed on one of them, $h / B$ is a function $g$ depending on the scaling variable $x=B^{2 / 3} P^{1 / 3} A^{-1}\left[\exp \left(A w_{\mathrm{c}}\right.\right.$ $\left.k_{\mathrm{B}} T-\exp \left(A w / k_{\mathrm{B}} T\right)\right]$ where $A w_{\mathrm{c}}=O\left(k_{\mathrm{B}} T\right)$ is a critical binding energy at the discontinuous transition to a completely collapsed state, $A$ is the size of a monomer, and $B$ is the distance between the walls. Equation 17 is derived by assuming $g(x)$ is a power law within a certain regime before the collapse $\left(w_{0}<w<w_{\mathrm{c}}\right)$, where $h$ is independent of $B$. 